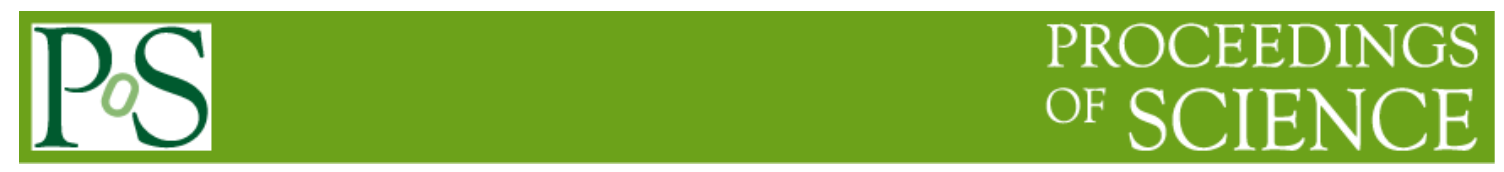

\title{
Spherically symmetric solution in Møller gravity
}

\section{Edward Rakhmetov ${ }^{1}$}

Skobeltsyn Institute of Nuclear Physics, M.V. Lomonosov Moscow State University

1(2), Leninskie gory, GSP-1, Moscow 119991, Russian Federation

E-mail: dsparelemail.ru

\section{Sergey Keyzerov}

Skobeltsyn Institute of Nuclear Physics, M.V. Lomonosov Moscow State University

1(2), Leninskie gory, GSP-1, Moscow 119991, Russian Federation

E-mail: errarewww-hep.sinp.msu.ru

In this paper, following C. Møller [1,2], we obtain spherically symmetric solution in the tetrad Møller gravity. We use this solution and the so-called PPN-formalism [3,5] to compare our theory with experiment and to improve restrictions on the theory constants.

The XXI International Workshop High Energy Physics and Quantum Field Theory June 23 - June 30, 2013

Saint Petersburg Area, Russia

Speaker 


\section{Introduction}

Spherically symmetric solutions are very important to understand the slow-motion and weak-field limit of the gravity theory under study and to confront this theory with Solar System experiments [4]. For example, the three classical tests of General Relativity are based on the spherically symmetric Schwarzschild solution and the weak-field limit.

In this paper we consider a spherically symmetric solution, which can be obtained in the interesting generalization of General Relativity suggested by C. Møller [1]. It is convenient to use in such analyses so-called PPN-formalism, specially built to study the slow-motion and weak-field limit of the extended theories of gravity [3].

Save for this little introduction and a short conclusion, this paper is organized as follows. In $\S 2$, we give a brief review of the Møller gravity. In $\S 3$, we obtain the spherically symmetric solution. In $\S 4$, we gain restrictions on the theory parameters, using PPN-formalism and data of Solar System experiments.

\section{Møller gravity review}

It was C. Møller, who first put forward this theory in 1978 [1]. The Møller gravity theory is a metric theory, in which the metric tensor

$$
g_{\mu \nu} \equiv \sum_{\alpha=0}^{3} \delta(\alpha \alpha) g(\alpha)_{\mu} g(\alpha)_{v} \equiv g(\alpha)_{\mu} g(\alpha)_{v}, \quad \delta(\alpha \beta) \equiv \operatorname{diag}\{-1,1,1,1\}
$$

is constructed from an orthonormal tetrad:

$$
g(\alpha)_{\mu} g(\beta)^{\mu} \equiv \delta(\alpha \beta)
$$

Here the indices in the brackets are frame (vielbein) indices, which run from 0 to 3 , and the summation over the repeated indices is implied. The stress tensor for this vector fields (denoted by $\left.f(\alpha)_{\mu v}\right)$ is, as usually:

$$
f(\alpha)_{\mu v} \equiv \partial_{[\mu} g(\alpha)_{v]}
$$

Where the antisymmetrization over the indices in the square brackets is implied. The coordinate indices can be turned into frame (vielbein) indices as it is presented below:

$C_{\mu \nu}(\alpha) g(\alpha)_{\lambda} \equiv C_{\mu \nu \lambda}$

We can obtain the Riche tensor from the stress tensor:

$$
\begin{aligned}
& R(\alpha \beta)=\frac{1}{2} \partial(\alpha) f(\mu \mu \beta)+\frac{1}{2} \partial(\beta) f(\mu \mu \alpha)+\frac{1}{2} \partial(\mu) f(\beta \alpha \mu) \\
& +\frac{1}{2} \partial(\mu) f(\alpha \beta \mu)-\frac{1}{2} f(\alpha \beta \mu) f(\nu \vee \mu)-\frac{1}{2} f(\beta \alpha \mu) f(\nu \nu \mu)+ \\
& -\frac{1}{2} f(\mu \nu \alpha) f(\nu \mu \beta)-\frac{1}{2} f(\mu \nu \alpha) f(\mu \nu \beta)+\frac{1}{4} f(\alpha \mu \nu) f(\beta \mu \nu)
\end{aligned}
$$

Where $\partial(\alpha) \equiv g(\alpha)^{\mu} \partial_{\mu}$

Contacting indices in different ways, we can build from the stress tensor three different scalars: 


$$
L_{1} \equiv f_{\alpha \beta \gamma} f^{\alpha \beta \gamma} L_{2} \equiv f_{\alpha \beta \gamma} f^{\beta \alpha \gamma} L_{3} \equiv f^{\alpha}{ }_{\alpha \gamma} f_{\beta}^{\beta \gamma}
$$

Then, in general case, the simplest action quadratic in the partial derivatives is:

$S=\int_{X}\left(k_{0}+k_{1} L_{1}+k_{2} L_{2}+k_{3} L_{3}\right) \sqrt{g} d x$

where $k_{0}, k_{1}, k_{2}, k_{3}$ are arbitrary dimensional constants.

Using (2.5) we can obtain more usual expression for Einstein-Møller action (up to a complete divergence):

$S=\frac{1}{2 \kappa} \int_{X}\left\{\Lambda+R+k_{1}^{\prime} L_{1}+k_{2}^{\prime} L_{2}\right\} \sqrt{g} d x$

We could not write the action with the terms $L_{1}$ and $L_{2}$, if we used just the metric tensor without the vielbein formalism. Thus, we have a generalization of metric gravity.

As we can see, Møller gravity theory coincides with General Relativity, if $k_{1}^{\prime}, k_{2}^{\prime}$ is equal to 0 .

Denoting the variation of the action with respect to $g(\mu)_{\alpha}$ as

$X(\mu)^{\alpha} \equiv \frac{1}{\sqrt{g}} \frac{\delta S}{\delta g(\mu)_{\alpha}}$,

we can write the symmetric and the antisymmetric parts of the equations of motion $X(\mu)^{\alpha} \equiv \frac{1}{\sqrt{g}} \frac{\delta S}{\delta g(\mu)_{\alpha}}=0$ separately. The symmetric part gives:

$X^{(\alpha \beta)}=-4 k_{3}\left(R^{\alpha \beta}-\frac{1}{2} g^{\alpha \beta}(R+\Lambda) \frac{\dot{H}_{j}}{\mathrm{j}}+2\left(2 k_{1}^{\prime}+k_{2}^{\prime}\right) \nabla_{\mu} f^{(\alpha \beta) \mu}-\left(2 k_{1}^{\prime}-k_{2}^{\prime}\right) f^{[\mu \nu] \alpha} f_{[\mu v]}^{\beta}+\right.$

$+\left(2 k_{1}^{\prime}+k_{2}^{\prime}\right) f^{\mu \nu(\alpha} f_{\mu \nu}^{\beta)}-2 k_{2}^{\prime} f^{\alpha}{ }_{\mu \nu} f^{\beta \mu \nu}+2 g^{\alpha \beta}\left(k_{1}^{\prime} f^{\mu \nu \lambda} f_{\mu \nu \lambda}+k_{2}^{\prime} f^{\mu \nu \lambda} f_{v \mu \lambda}\right)=0$

The antisymmetric part of the equations of motion is

$X^{[\alpha \beta]}=2\left(2 k_{1}^{\prime}-k_{2}^{\prime}\right) \nabla_{\mu} f^{[\alpha \beta] \mu}-4 k_{2}^{\prime} \nabla_{\mu} f^{\mu \alpha \beta}-\left(2 k_{1}^{\prime}-3 k_{2}^{\prime}\right) f^{\mu v[\alpha} f_{\mu \nu}^{\beta]}=0$

If $k_{1}^{\prime}, k_{2}^{\prime}$ are equal to zero, the antisymmetric part vanishes. The symmetric part of the equations of motion gives us General Relativity. As we can see, in the Møller gravity theory we have more restrictions on frame vectors, than in General Relativity, because of the additional antisymmetric part of the equations of motion. And now the action of the theory is not invariant under the rotations of the frame, unlike in General Relativity.

\section{Spherically symmetric solution}

Let us write the required spherically symmetric metric using isotropic coordinates [4]:

$$
d s^{2}=e^{2 \gamma(r)} d t^{2}-e^{2 \alpha(r)}\left\{d r^{2}+r^{2}\left(d \theta^{2}+\sin ^{2} \theta d \varphi^{2}\right)\right\}
$$

Then the metric tensor is:

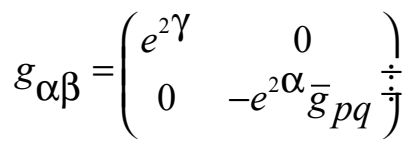


It is convenient to write an ansatz for the frame:

$g(0)_{0}=e^{\gamma}, g(0)^{0}=e^{-\gamma}, g(a)_{0}=0, g(a)_{q}=e^{\alpha} \bar{g}(a)_{q}, g(a)^{q}=-e^{-\alpha} \bar{g}(a)^{q}$

Where

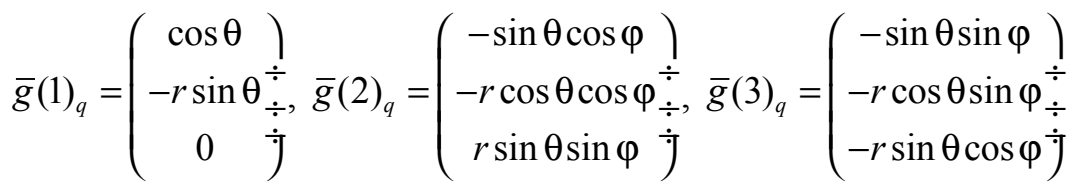

is an orthonormal frame in 3-dimensional flat space.

Using (3.1)-(3.4) and the equations of motion, after some calculations we obtain three equations for two functions parameterizing the metric and the frame:

$$
\begin{aligned}
& 2 \alpha_{, r r}+\alpha_{, r}^{2}+4 r^{-1} \alpha_{, r}=\xi\left(2 \alpha_{, r}^{2}-2 \gamma_{, r r}-\gamma_{, r}^{2}-2 \alpha_{, r} \gamma_{, r}-4 r^{-1} \gamma_{, r}\right) \\
& \alpha_{, r}^{2}+2 r^{-1} \alpha_{, r}+2 \gamma_{, r} \alpha_{, r}+2 r^{-1} \gamma_{, r}=-\xi\left(2 \alpha_{, r}^{2}+\gamma_{, r}^{2}+4 r^{-1} \alpha_{, r}\right) \\
& \alpha_{, r r}+r^{-1} \alpha_{, r}+r^{-1} \gamma_{, r}+\gamma_{, r r}+\gamma_{, r}^{2}=\xi\left(-2 \alpha_{, r r}+\gamma_{, r}^{2}-2 \alpha_{, r} \gamma_{, r}-2 r^{-1} \alpha_{, r}\right)
\end{aligned}
$$

Where $\xi \equiv 2 k_{1}^{\prime}+k_{2}^{\prime}$

Obviously, this system is overdetermined. Nevertheless, it has a solution in the form of

$$
\begin{aligned}
& \alpha=A \ln \left(1-r_{0} / r\right)+B \ln \left(1-r_{1} / r\right) \\
& \gamma=C \ln \left(1-r_{0} / r\right)+D \ln \left(1-r_{1} / r\right)
\end{aligned}
$$

Where

$$
\begin{aligned}
& A=\frac{1-\xi+\sqrt{(1-2 \xi)(1+\xi)}}{1-3 \xi}, B=\frac{1-\xi-\sqrt{(1-2 \xi)(1+\xi)}}{1-3 \xi}, \\
& C=-\frac{2 \xi+\sqrt{(1-2 \xi)(1+\xi)}}{1-3 \xi}, D=\frac{\sqrt{(1-2 \xi)(1+\xi)}-2 \xi}{1-3 \xi} \\
& r_{0}=\frac{1}{1+2 \xi}\left(\xi-\frac{\sqrt{(1-2 \xi)(1+\xi)}}{2} \dot{\div} \overline{\dot{r}}, r_{1}=\frac{1}{1+2 \xi}\left(\xi+\frac{\sqrt{(1-2 \xi)(1+\xi)}}{2} \dot{\div} \overline{\dot{r}}\right.\right.
\end{aligned}
$$

And where $\bar{r}$ is the Schwarzschild radius.

Thus the spherically symmetric metric looks like:

$$
d s^{2}=\left(1-r_{0} / r\right)^{2 C}\left(1-r_{1} / r\right)^{2 D} d t^{2}-\left(1-r_{0} / r\right)^{2 A}\left(1-r_{1} / r\right)^{2 B}\left(d x^{2}+d y^{2}+d z^{2}\right)
$$

As we can see, the spherically symmetric solution coincides with Schwarzschild metric not only in the trivial case, when our theory coincides with General Relativity and $k_{1}^{\prime}, k_{2}^{\prime}$ are equal to zero separately, but in the case when $\xi \equiv 2 k_{1}^{\prime}+k_{2}^{\prime}=0$ as well.

Up to the second order in inverse radius we obtain for the space-time metric

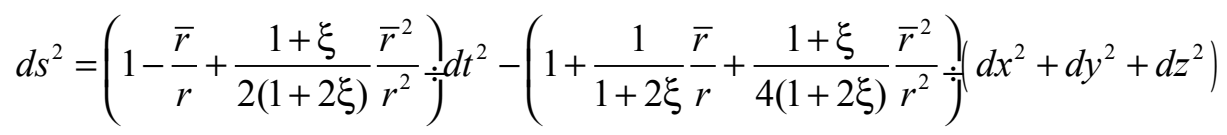

It is convenient to use this expression in the so-called PPN-formalism to compare our theory with experiment. 


\section{Spherically symmetric solution and the PPN-formalism}

The main idea of the parameterized post-Newtonian formalism is that the comparison of metric theories of gravity with experiment becomes simple when we use the weak-field limit. This approximation is known as the post-Newtonian limit [3,4]. It is sufficiently accurate to the most solar-system tests [5]. In this approximation the space-time metric predicted by nearly every metric theory of gravity has the same structure. The space-time metric can be written as an expansion about the Minkowski metric in terms of dimensionless gravitational potentials of varying degrees of smallness:

$g_{00}=1-2 U+2 \beta U^{2}+\ldots, g_{i j}=-(1+2 \gamma U) \delta_{i j}+\ldots$

that are constructed from the matter variables in imitation of the Newtonian gravitational potential $[3,5]$. For example, for the spherically symmetric distribution of matter

$U=\int \frac{\rho(\mathbf{y})}{|\mathbf{x}-\mathbf{y}|} d^{3} \mathbf{y}=\frac{m}{r}, \quad r \equiv|\mathbf{x}|$

Where $\rho(\mathbf{y})$ is the density of rest mass as measured in a local freely falling frame momentarily commoving with the gravitating matter.

The only way that one metric theory differs from another is in the numerical values of the coefficients that appear in front of the metric potentials. The PPN-formalism inserts parameters in place of these coefficients. In such a way parameters values depend on the theory under study [3].

Comparing (3.14), (4.1) and (4.2), we obtain PPN-parameters $\beta$ and $\gamma$ for Møller gravity theory: $\gamma=\frac{1}{1+2 \xi}, \beta=\frac{1+\xi}{1+2 \xi}$, where $\xi \equiv 2 k_{1}^{\prime}+k_{2}^{\prime}$

The parameters $\beta$ and $\gamma$ are the well-known Eddington-Robertson-Schiff parameters used to describe the classical tests of General Relativity [3]. Recently tests of PPN-parameters $\beta$ and $\gamma$ have reached high precision, including the light deflection, the perihelion advance of Mercury and the Shapiro time delay [5]. Time delay tests give the best estimation for $\gamma[5,6]$ with a result $\gamma-1=(2.1 \pm 2.3) \times 10^{-5}$

From (4.3) and (4.4) it follows that $\xi \equiv 2 k_{1}^{\prime}+k_{2}^{\prime}=(-1.1 \pm 1.2) \times 10^{-5}$

As we can see, the combination $\xi \equiv 2 k_{1}^{\prime}+k_{2}^{\prime}$ of the theory parameters has to be very small to be in agreement with experimental data. This is the only restriction on the theory parameters we can get from the spherically symmetric solution and data of the solar-system experiments.

\section{Conclusions}

In $\S 2$ we present the spherically symmetric solution of Møller gravity in an explicit form. As we can see, the spherically symmetric solution appears not only in the case, when the additional constants of the Einstein-Møller action are small, as it was shown by Møller [1], but also in the case of arbitrary constants too.

The peculiar moment is that the spherically symmetric solution coincides with Schwarzschild metric not only in the trivial case, when our theory coincides with General 
Relativity (when $k_{1}^{\prime}, k_{2}^{\prime}$ are equal to zero separately), but in the case when $\xi \equiv 2 k_{1}^{\prime}+k_{2}^{\prime}=0$ as well.

The combination $\xi \equiv 2 k_{1}^{\prime}+k_{2}^{\prime}$ of the theory parameters has to be very small (of the order of $10^{-5}$ ) to be in agreement with experimental data. This is the only restriction on the theory parameters we can get from the spherically symmetric solution and data of the solar-system experiments.

\section{Acknowledgements}

The authors are grateful to E.E. Boos, I.P. Volobuev, M.N. Smolyakov for valuable discussions. The work was supported by grant of Russian Ministry of Education and Science NS-3042.2014.2.

\section{References}

[1] C. Møller, On the Crisis of Gravitation and a Possible Solution, Mat. Fys. Medd. Dan. Vid. Selsk., v.39 n.13, 1978.

[2] H.-J Treder, C. Møller, et al, Einstein-centinarium, (miscellany) Akademie-Verlag, Berlin, 1979

[3] Clifford M. Will, Theory and experiment in gravitational physics, Cambridge University Press, 1981.

[4] S. Capozziello, V. Faraoni, Beyond Einstein Gravity, Springer Science, 2011.

[5] Clifford M. Will, The Confrontation between General Relativity and Experiment, Living Reviews in Relativity, 9, 2006.

[6] B. Bertotti, L. Iess, P. Tortora, A test of general relativity using radio links with the Cassini spacecraft, Nature, 425, 374-376, 2003. 